\title{
ANISOTROPY OF PHONON EMISSION FROM HOT ELECTRONS IN GERMANIUM
}

\author{
W. Reupert, K. Lassmann, and P. de Groot \\ Universität Stuttgart, Physikalisches Institut \\ Teilinstitut 1, 7 Stuttgart 80 , Germany
}

We have measured quantitatively the anisotropy of the electron acoustical phonon scattering in n-germanium in five directions of the (1TO) plane utilizing tunneling junctions for calibrated detection of the phonon radiation emitted from a small avalanche breakdown region in the germanium surface. For comparison and evaluation of phonon focusing a constantan heater and a tunneling junction were also used as phonon sources. A similar experiment has been reported by A. Zylbersztejn $/ 1 /$, but he could only compare the ratio of the amplitudes of longitudinal and transverse phonon pulses in a given crystal direction to the calculation.

Our sample was doped with $\mathrm{Sb}\left[3 \times 10^{14} \mathrm{~cm}^{-3}\right]$, the schematic arrangement is shown in Fig.1. The avalanche heater consisted of two alloyed $n^{+} n$ contacts $0.5 \mathrm{~mm}$ wide. The signals were generated by current pulses in the [001] direction. This implies the same electron temperature $\mathrm{T}_{e}$ and the same electron density in each valley of the conduction band. The Constantan film as well as the $\mathrm{Sn}_{-} \mathrm{SnO}_{\mathrm{x}}-\mathrm{Sn}$ tunneling junction had the same area as the avalanche heater. The sample was immersed into liquid helium at $2 \mathrm{~K}$. Current pulses of 0.4 us at a repetition rate of $4 \mathrm{kHz}$ were applied. The signals of the avalanche heater received by the tunneling junctions are shown in Fig. 1.

E. Conwell /2/ calculated the anisotrpy of the steady state phonon distribution for an isotropic many valley semiconductor. In our calculations we took into account the anisotropy of the sound velocity and evaluated the electron phonon matrix elements for the special case of phonon propagation in the (1T0) plane from interpolation formulas given by $C$. Herring and $E$. Vogt $/ 3 \%$. The contributions of the two transverse modes were calculated separately. To calculate the phonon rate detected by the junction we may write 


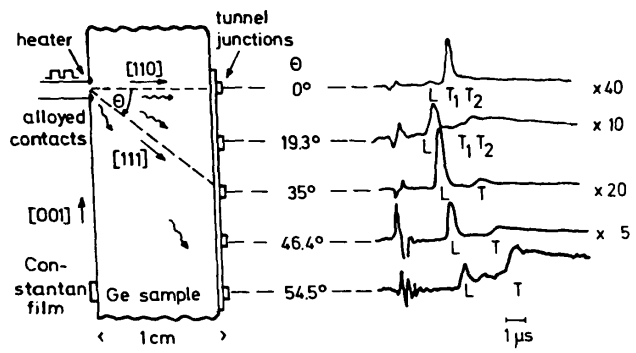

Fig. 1 Schematic sample arrangement and signals in 5 directions of the ( 1 \%) plane at the same input power. Lattice temperature 2K.

$$
\dot{\mathrm{N}}_{p}=\int_{2 \Delta}^{4 \Delta}\left(\left(\mathrm{N}_{q p}-\overline{\mathrm{N}}_{q p}\right) / \tau\right) \mathrm{z}(\varepsilon) \mathrm{d} \varepsilon,
$$

where $\mathrm{N}_{\mathrm{qp}}$ is the steady state number of phonons of polarization $\mathrm{p}$ and wave vector $q$ and $\hat{N}_{q p}$ thermal equilibrium number small compared to $\mathrm{N}_{\mathrm{gp}}$ at helium temperatures. $\mathrm{Z}(\varepsilon)$ is the phonon density of states. The relaxation time $r$ was identified with the signal rise time of about .2 us (the response time of the receiving tunneling junctions is much faster at $2 \mathrm{~K}$ ). The lower limit of integration is given by the spectral sensitivity of the tunneling junction ( $2 \Delta$ is the energy gep of $t i n)$. For electron temperatures below $T_{e}=100 \mathrm{~K}$ we found that the number of phonons with energy $\varepsilon>4 \Delta$ is negligible. The tunneling junction operates as a phonon counter in this energy interval.

The normalized signals of the Constantan heater and the tunnelling junction were compared to the theoretical values for an isotropic phonon source taking into account the geometrical factors and phonon focusing values for $\operatorname{LiF} / 4 /$, which should be valid for Ge within a few percent. We found good agrement and took the measured focusing factors to eliminate phonon focusing from the signals of the avalanche heater. In the saturation range of the avalanche breakdown, when the donors are ionized, the kinetic energy of the hot electrons can be estimated from the ionization energy of the $\mathrm{Sb}$ donors $(9.7 \mathrm{meV})$ corresponding to $75 \mathrm{~K}$. Fig. 2 shows the good agreement of the calculated phonon distribution for $T_{e}=75 \mathrm{~K}$ with measured values in the 

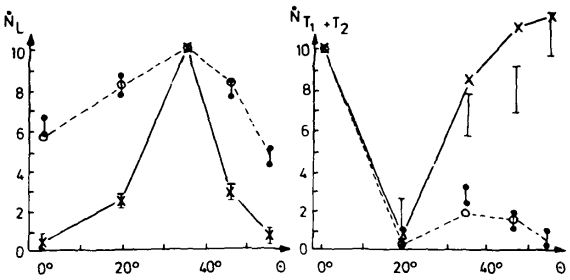

Fig.2 Relative rates of phonons received at the tunnel junctions. The longitudinal (L) pulses are normalized in [111], the transverse $\left(T_{1}+T_{2}\right)$ in $[110]$. Heat pulse measured $(\boldsymbol{l})$, electron-phonon interaction measured (I), and calculated $(+)$.

saturation range. In accordance to theory the anisotropy of the phonon distribution increased with decreasing input power (thet means decreasing $T_{e}$ ). At the beginning of the avalanche breakdown we estimate from experiment $\mathrm{T}_{e}=30 \mathrm{~K}$. To estimate the electron temperature we evaluated the longitudinal phonon signals because of a background signal of the transverse phonons rapidly increasing with growing input power of the avalanche heater. This background was not seen at corresponding powers of the Constantan heater and may be a property of the avalanche heater.

\section{References:}

11/ A.zylbersztejn, Proc. 10 th int. Conf. Semiconductors p 134 (70)

12/ E. Conwell, Phys. Rev. 135, A814 (64)

$13 /$ C. Herring and E. Vogt, Phys. Rev. 101, 944 (56)

14 / B.Taylor, H.J.Maris, and C.Elbaum, Phys. Rev. B3, 1462 (71) 